\title{
An Enhanced Ultrasonic Frequency Closed-Loop Control System for Biological Decomposition
}

\author{
E. Shi, C. Hu, Y.S. Xu \\ College of Physics, Optoelectronics and Energy \\ Soochow University \\ China
}

\begin{abstract}
Frequency automatic tracking is a main characteristic of ultrasonic power system, especially in the field of biological decomposition. In this paper, after analysing characteristics of multiple peaks of transducer, a reliable power control and frequency tracking strategy is introduced. It is based on an accurate and fast strategy of frequency tracking; by taking samples of the error of phase difference with small resistance method, two control strategies of DDS (Direct Digital Synthesizer) and PI (proportional-integral) adjustment associate with each other to reduce the error. Then the transducer array keeps an effective working state, the whole system realizes low distortion, variable resonance frequencies and adjustable amplitude. The system improves power factor and meets the needs for dealing with different kinds of biological samples.
\end{abstract}

Keywords- frequency tracking; DDS; PI control; biological decomposition

\section{INTRODUCTION}

In the process of biological decomposition, cells will be cleaved by ultrasonic cavitation effect; important biological materials such as protein will be obtained. But changes of properties in samples bring variable parameters, for instance, temperature and intensity, which influence the effect of biological decomposition. If ultrasonic power supply can't adapt the changes, vibration system will work in a nonresonant status and the disharmonic status will lead to a decrease of amplitude of vibration. To improve the power factor, it is required that ultrasonic power supply and the load of ultrasonic transducer should work in resonate state and whole system have a maximum energy output [1]. So a closedloop control strategy should be added to maintain the stability of biological decomposition. In the field of ultrasonic power supply, analogue chips are common used in PWM control, such as TL494, SG3525. Besides, a direct use of PWM wave produced by microcontroller is applied to drive vibration system by power inverter. The two ways have limitations: the former one has a slow dynamic response, inconvenient parameter adjustment and a severe drift of resonance frequency; the latter one will not satisfy the demand of accurate regulation because it has a narrow frequency resolution limited by working frequency of microcontroller. In this paper, based on characteristics of frequency of transducer, DDS technology associated with PI adjustment is applied in the precise control of resonance frequency of the whole system, vibration amplitude is controllable by a set of D/A output and power is improved by $8 \% \sim 12 \%$.

\section{SYSTEM COMPONENTS OF HARDWARE}

As shown in figure 1, the ultrasonic system for biological decomposition consists of amplitude regulating circuit, a high frequency inverter unit, and an impedance matching unit and feedback network.

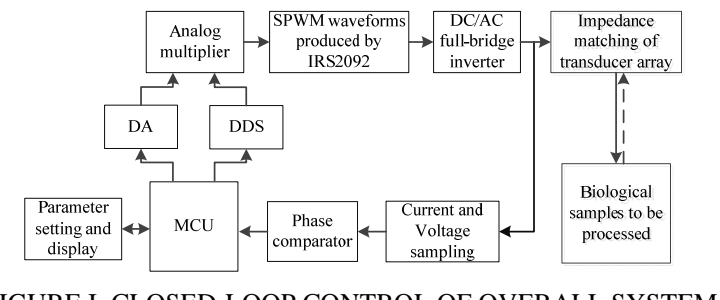

FIGURE I. CLOSED-LOOP CONTROL OF OVERALL SYSTEM.

When biological samples is being processed, rising temperature and changing intensity give a reaction back to transducer array that shifts the resonance frequency. When the system starts to work, frequency and phase difference are acquired to check the state of transducer array. By current and voltage sampling and phase comparison, the sign and value of phase difference is obtained by a microcontroller of ATmega16 with A/D sampling. According to it, ultrasonic power supply shifts the driving frequency in a corresponding step and direction to keep pace with alteration of frequency of transducer array.

Precise amplitude control is vital in biological decomposition because the effect is influenced by different power distribution. So a digital to analog conversion chip of DAC7512 is applied to control the amplitude by changing the 12-bits input current control word, then output of DAC7512 is multiplied by the output of DDS chip of AD9850. Controlled by DDS, DC-AC inverter will be controlled in digital natural sampling based on SPWM (Sinusoidal PWM) [2]; The D class amplifier chip of IRS2092 is used to generate two SPWM waves which regulate the on-off of four MOSFET power transistors. The two complementary SPWM waves control the full-bridge power transistors to alternate breakover. By filtering in the following level, the output signal has the same frequency of the DDS chip of AD9850 and it can drive the load of transducer array. What's more, adding dead-time circuit whose dead time can be set from 25 ns to 105 ns in the chip of IRS2092 will avoid the influence about the delay of turn-off of MOSFET power transistors. Due to larger static capacitance, transducer array shows great capacity; if alternating voltage is added directly, low power factor will 
exist. For capacitive load, status of pure resistance is achieved by connecting compensating inductance with the load in series.

\section{FEedBACK Signal SAMPLING AND FREQUENCY TRACKING}

A. Characteristic of Multiple Peaks of Resonance Frequency in Transducer

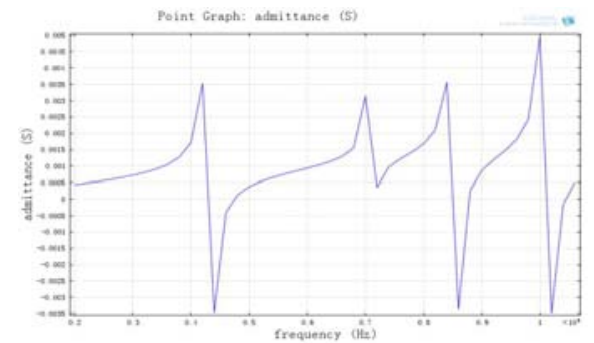

FIGURE II. CHARACTERISTIC OF MULTIPLE PEAKS OF TRANSDUCER

To know the characteristics of resonance frequency of transducer, finite element analysis software of Comsol Multiphysics is applied to analyze the relationship between admittance and frequency of transducer. According to the relationship between impedance and admittance, the higher the admittance is, the lower the impedance will be. The figure 2 clearly shows that there are several points of peaks which are distributed among a number of frequencies [3].

Research shows that taking control of two parameters of phase difference and current value [4] is an effective way to search and track resonance frequency. This way searches for the maximum current point of transducer and zero-phasedifference point with a certain strategy, then locks coincidence of the two parameters. Obviously, strategy of control is complex and there exist a lot of uncertain factors. Usually, when a transducer is produced, range of resonance frequency is known. To ensure the stability of power supply and reduce uncertain factors, range of frequency tracking will be divided into several parts so that each part contains only one resonance frequency, then it is simple to automatically track of frequency without searching for each resonance frequency.

\section{B. Principle of Taking Sample of Phase Difference}

Only by sampling the signal of both current and voltage can make the acquisition the present state of transducer array. The small resistance method is chosen in the experimental stage. As shown in figure 3 , adding a $1 \mathrm{ohm}$ precise noninductive resistance in the output circuit, the voltage of both ends of the resistance represents the current of transducer array. In order to ensure the accuracy of measurement, such a small signal also should be amplified in the next level.

The small signal with same frequency and amplitude of current and voltage will be compared by high-speed op-amp; the sign of phase difference acquired after zero-crossing circuit indicates which pulls ahead between voltage and current. A band-pass filter is designed to wipe out interference signal and maintain signal waveform. The depth of frequency tracking adjustment relies on the value of phase difference. So an analogue multiplier of AD633 chip is applied to measure phase difference; Current is represented by $X(\mathrm{t})$, voltage is represented by $Y(\mathrm{t})$.

$$
X(t)=U_{m} \sin \left(\omega t-\phi_{1}\right), Y(t)=U_{n} \sin \left(\omega t-\phi_{2}\right)
$$

In the process of biological decomposition, cells will be cleaved by ultrasonic cavitation effect; important biological materials such as protein will be obtained. But changes of properties in samples bring variable parameters, for instance, temperature and intensity, which influence the effect of biological decomposition. If ultrasonic power supply can't adapt the changes, vibration system will work in a nonresonant status and the disharmonic status will lead to a decrease of amplitude of vibration. To improve the power factor, it is required that ultrasonic power supply and the load of ultrasonic transducer should work in resonate state and whole system have a maximum energy output [1]. So a closedloop control strategy should be added to maintain the stability of biological decomposition. In the field of ultrasonic power supply, analogue chips are common used in PWM control, such as TL494, SG3525. Besides, a direct use of PWM wave produced by microcontroller is applied to drive vibration system by power inverter. The two ways have limitations: the former one has a slow dynamic response, inconvenient parameter adjustment and a severe drift of resonance frequency; the latter one will not satisfy the demand of accurate regulation because it has a narrow frequency resolution limited by working frequency of microcontroller. In this paper, based on characteristics of frequency of transducer, DDS technology associated with PI adjustment is applied in the precise control of resonance frequency of the whole system, vibration amplitude is controllable by a set of $\mathrm{D} / \mathrm{A}$ output and power is improved by $8 \% \sim 12 \%$.

As shown in figure 1 , the ultrasonic system for biological decomposition consists of amplitude regulating circuit, a high frequency inverter unit, and an impedance matching unit and feedback network.

When biological samples is being processed, rising temperature and changing intensity give a reaction back to transducer array that shifts the resonance frequency. When the system starts to work, frequency and phase difference are acquired to check the state of transducer array. By current and voltage sampling and phase comparison, the sign and value of phase difference is obtained by a microcontroller of ATmega16 with A/D sampling. According to it, ultrasonic power supply shifts the driving frequency in a corresponding step and direction to keep pace with alteration of frequency of transducer array.

Precise amplitude control is vital in biological decomposition because the effect is influenced by different power distribution. So a digital to analog conversion chip of DAC7512 is applied to control the amplitude by changing the 12-bits input current control word, then output of DAC7512 is multiplied by the output of DDS chip of AD9850. Controlled by DDS, DC-AC inverter will be controlled in digital natural sampling based on SPWM (Sinusoidal PWM) [2]; The D class amplifier chip of IRS2092 is used to generate two SPWM waves which regulate the on-off of four MOSFET power 
transistors. The two complementary SPWM waves control the full-bridge power transistors to alternate breakover. By filtering in the following level, the output signal has the same frequency of the DDS chip of AD9850 and it can drive the load of transducer array. What's more, adding dead-time circuit whose dead time can be set from 25 ns to 105 ns in the chip of IRS2092 will avoid the influence about the delay of turn-off of MOSFET power transistors. Due to larger static capacitance, transducer array shows great capacity; if alternating voltage is added directly, low power factor will exist. For capacitive load, status of pure resistance is achieved by connecting compensating inductance with the load in series.

To know the characteristics of resonance frequency of transducer, finite element analysis software of Comsol Multiphysics is applied to analyze the relationship between admittance and frequency of transducer. According to the relationship between impedance and admittance, the higher the admittance is, the lower the impedance will be. The figure 2 clearly shows that there are several points of peaks which are distributed among a number of frequencies [3].

Research shows that taking control of two parameters of phase difference and current value [4] is an effective way to search and track resonance frequency. This way searches for the maximum current point of transducer and zero-phasedifference point with a certain strategy, then locks coincidence of the two parameters. Obviously, strategy of control is complex and there exist a lot of uncertain factors. Usually, when a transducer is produced, range of resonance frequency is known. To ensure the stability of power supply and reduce uncertain factors, range of frequency tracking will be divided into several parts so that each part contains only one resonance frequency, then it is simple to automatically track of frequency without searching for each resonance frequency.

Only by sampling the signal of both current and voltage can make the acquisition the present state of transducer array. The small resistance method is chosen in the experimental stage. As shown in figure 3 , adding a 1 ohm precise noninductive resistance in the output circuit, the voltage of both ends of the resistance represents the current of transducer array. In order to ensure the accuracy of measurement, such a small signal also should be amplified in the next level.

The small signal with same frequency and amplitude of current and voltage will be compared by high-speed op-amp; the sign of phase difference acquired after zero-crossing circuit indicates which pulls ahead between voltage and current. A band-pass filter is designed to wipe out interference signal and maintain signal waveform. The depth of frequency tracking adjustment relies on the value of phase difference. So an analogue multiplier of AD633 chip is applied to measure phase difference; Current is represented by $X(\mathrm{t})$, voltage is represented by $Y(\mathrm{t})$.

$$
Z=X Y=-1 / 2 U_{m} U_{n}\left\{\cos \left(2 \omega t-\left(\phi_{1}+\phi_{2}\right)\right)-\cos \left(\phi_{2}-\phi_{1}\right)\right\}
$$

After filtering out AC component, voltage represents phase difference is:

$$
U(t)=1 / 2 U_{m} U_{n} \cos \left(\phi_{2}-\phi_{1}\right)
$$

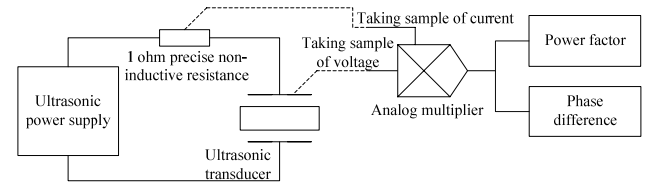

FIGURE III. ACQUISITION OF PHASE DIFFERENCE.

By differential amplification and $\mathrm{A} / \mathrm{D}$ sampling, phase difference of voltage and current $\sigma=\left|\varphi_{1}-\varphi_{2}\right|$ is obtained according to the results of multiply and the power can be calculated below; Where, $U$ and $I$ are effective value.

$$
P=U I \cos \sigma
$$

\section{Accurate Control of DDS}

DDS is a complete direct digital frequency synthesizer, it has the advantages of high phase resolution, wide range of frequency, swift switching frequency, low power consumption and programmable. The chip of AD9850 changes output frequency by changing frequency control word and incremental extent phase. When the external clock produces a clock signal, the phase accumulator will add the accumulation of phase which is superimposed together with incremental extent phase. The result of the addition is taken as the address to search the address of the waveform ROM table in the amplitude algorithm, and find out quantitative range code value matched by the unit phase in the ROM table. Then D/A converter turns quantitative range code value into analogue quantity and the output signal is finally formed after the smooth of low-pass filter [5]. The output frequency $f_{0}$ can be calculated by

$$
f_{0}=M f_{s} / 2^{N}
$$

Where, $\mathrm{M}$ is value of frequency word, $\mathrm{N}$ is the length of frequency control word, $f_{\mathrm{s}}$ is the frequency of reference clock and its maximum value is $180 \mathrm{MHz}$. A relatively low frequency brings relatively large amplitude, low heat but low impact acceleration while a relatively high frequency will follow with large impact acceleration but low amplitude. To satisfy this condition, DDS module should shift from 10 40 kHz. Output signal will go through driving circuit and control full-bridge inverter by regulating on-off of MOSFET power transistors.

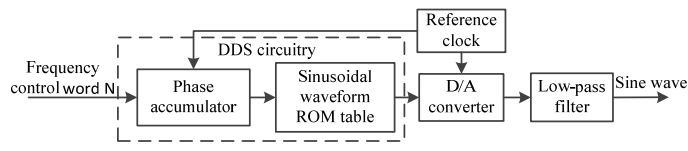

FIGURE IV. FUNCTIONAL BLOCKS OF DDS. 
D. Strategy of Frequency Tracking

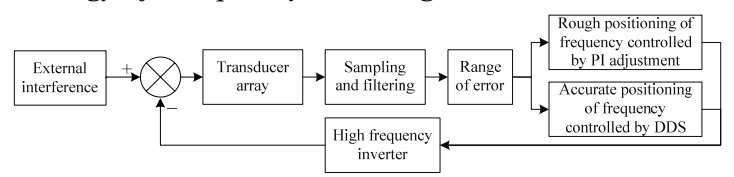

FIGURE V. BLOCK DIAGRAM OF AUTOMATIC FREQUENCY TRACKING.

In the block diagram of figure 5 , influenced by external interference, state of transducer array will be changed. If the error of change is small or the error is changed rapidly, rough positioning of frequency controlled by PI adjustment realizes its function of fast locking of range of resonance frequency. After preliminary optimization of PI adjustment, the error gets smaller, then accurate positioning of frequency controlled by DDS starts to take effect to track the changing frequency of transducer array further. After high frequency inverter, the system maintains a dynamic balance between external interference and adjusted output frequency of frequency tracking module.

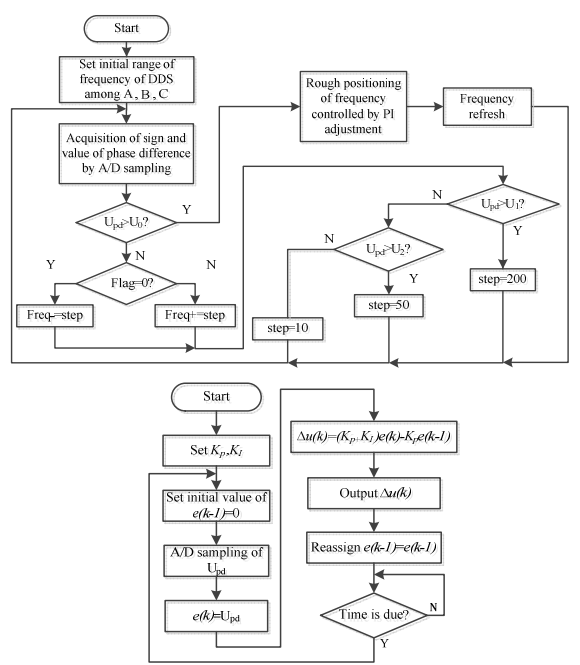

FIGURE VI. FLOW CHART OF FREQUENCY TRACKING.

Based on the characteristics of multiple peaks in transducer, the range of frequency is set into three parts: range $\mathrm{A}$ is from $10-20 \mathrm{kHz}$, range $\mathrm{B}$ is from $20-30 \mathrm{kHz}$, range $\mathrm{C}$ is from $30-40$ $\mathrm{kHz}$. The circumstances of several resonance frequencies in a range will be effectively reduced and the tracking time is greatly saved. $U_{p d}$ is voltage represents the value of phase difference between current and voltage acquired from transducer array; Flag is the sign of phase difference; $\mathrm{U}_{0}>\mathrm{U}_{1}>\mathrm{U}_{2}$, the three represent different accuracy of regulation by DDS.

In the accurate positioning of resonance frequency, if phase difference is lower than a certain threshold of $\mathrm{U}_{0}$, such circumstance will be handled by the rough positioning part. Particularly, the step has different spans from 10 to $200 \mathrm{~Hz}$ according to voltage represents the value of phase difference from $U_{0}$ to $U_{2}$ so that the module can tightly keep pace with changes of resonance frequencies of transducer array [6]. As shown in left part of figure 6 , if the sign of phase difference Flag $<0$, module adjusts the frequency towards the right side until phase difference decreases before the module is carried out in the next period of control. If frequency moves to left side, it follows the same principle as above. Freq is the frequency refreshed by DDS chip of AD9850 and a small step will reduce the speed of tracking and a large step will affect the quality of tracking, so a minimum step of $10 \mathrm{~Hz}$ is selected in the experiments.

In contrast with PID control, PI control cancels the differentiation term, which prevents the oscillation when the power supply is starting. System model of PI control is:

$$
u(\mathrm{t})=K_{p} e(\mathrm{t})+K_{I} \int_{0}^{t} e(\tau) d \tau
$$

Where, $K_{\mathrm{p}}$ is coefficient of proportionality, $K_{\mathrm{I}}$ is coefficient of integral, $e(t)$ is error signal. After simplifying it to difference equation:

$$
\Delta u(k)=u(k)-u(k-I)=\left(K_{p}+K_{I}\right) \mathrm{e}(k)-K_{P} \mathrm{e}(k-1)
$$

$U_{\text {pd }}$ is voltage represents the value of phase difference between current and voltage acquired by $\mathrm{A} / \mathrm{D}$ sampling, the error of $e(k)$ is obtained, by discrete digital PI calculation, adjusting values of $\Delta u(k)$ which will be converted to phase difference of $\sigma$ is acquired.

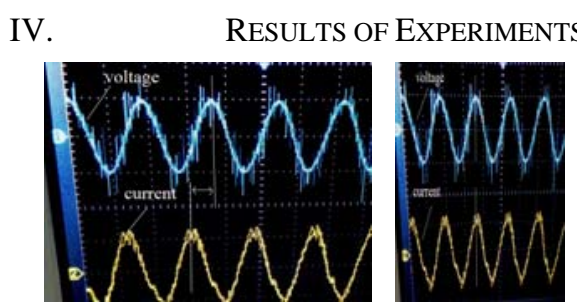

FIGURE VII. ULTRASONIC POWER SUPPLY HAS KEPT TRACKED ON THE WAVEFORM.

In figure 7 taken from oscilloscope, channel 1 is voltage signal and channel 2 is current signal sampled by the small resistance method. Both of the two parts are displayed after ten times the attenuation, the voltage is 5 volts per lattice, the current is $50 \mathrm{~mA}$ per lattice. In the left part, ultrasonic power supply is designed without the frequency tracking module and impedance match so that it will be compared with the one with the closed loop in the right part. Clearly shows in figure 7, the phase difference $\sigma$ is about $0.2 \pi$ and power factor is about 0.79. Owing to closed-loop control strategy and further impedance match, the phase difference is nearly close to zero, which means the ultrasonic system works in the resonance frequency and the power factor has been enhanced.

As shown in table 1, the frequency in ultrasonic power automatically tracked to $19.450 \mathrm{kHz}$, it has well realized the frequency tracking of the system. With the assist of impedance analyzer, the three parameters of frequency of transducer array, resonance frequency of transducer array and impedance matching circuit, working frequency of the whole system are measured in table 1 . 
TABLE I . RESULTS OF FREQUENCY TRACKING.

\begin{tabular}{|c|c|c|c|}
\hline & $\begin{array}{c}\text { Initial } \\
\text { state }\end{array}$ & $\begin{array}{c}\text { Without } \\
\text { frequency } \\
\text { tracking }\end{array}$ & $\begin{array}{c}\text { Frequency } \\
\text { tracking based } \\
\text { on PI and DDS }\end{array}$ \\
\hline $\begin{array}{c}\text { Frequency } \\
\text { X }\end{array}$ & $20 \mathrm{kHz}$ & $19.448 \mathrm{kHz}$ & $19.448 \mathrm{kHz}$ \\
\hline $\begin{array}{c}\text { Frequency } \\
\text { Y }\end{array}$ & $20 \mathrm{kHz}$ & $19.450 \mathrm{kHz}$ & $19.450 \mathrm{kHz}$ \\
\hline $\begin{array}{c}\text { Frequency } \\
\mathrm{Z}\end{array}$ & $20 \mathrm{kHz}$ & $20 \mathrm{kHz}$ & $19.450 \mathrm{kHz}$ \\
\hline $\begin{array}{c}\text { Power } \\
\text { factor }\end{array}$ & 1 & 0.79 & 0.91 \\
\hline
\end{tabular}

Experiments show that the compound control of PI and DDS is an effective strategy in frequency tracking. Ultrasonic closed-loop control system for biological decomposition shows its characteristics of real-time tracking and power factor is enhanced by $8 \% \sim 12 \%$ in the condition of same load and same input. The power system has a stable performance in frequency tracking and meets the requirements of design.

\section{ACKNOWLEDGEMENTS}

The research work was supported by Soochow University \& Jiemei Group United Key Laboratory Foundation of Biomedical Engineering Instruments under Grant No. JJ10800311.

\section{REFERENCES}

[1] Bing-yi Li, Zhe Ding, Research on the Frequency Automatic Tracking in Ultrasonic Power Supply Based on Fuzzy-DDS, Proceeding of the IEEE International Conference on Automation and Logistics, Zhengzhou, China, 2012.

[2] Chen Xiao-ping, Wang Nian-chun, Ma Yu-long, SPWM Control Technique Based On PIC single Chip Computer, Power Supply Technologies And Applications, Vol.9 No.3, Nanjing, China, PP.39-42, 2006.

[3] Huang Hongbin, Tang Yongjun, Fu Meirong, Chen Meng, Design of Automatic Frequency tracking System of Ultrasonic Power Supply Based on FPGA, Electric machining and die, Guangzhou, China, PP. 3235, 2013.

[4] Guan-qiang Dong, Lei Song, Zong-Xiao Yang, Research and application on the frequency automatic tracking of ultrasonic power based on DSP, Proceedings of the 2012 International Conference on Advanced Mechatronic Systems, Tokyo, Japan, PP. 478-481, 2012.

[5] Yuan Zongheng, Wei Changwei, The design of high-powerultrasonic power supply based on DDS, Power Electronics, Guiyang and Guilin, Vol.44 No.11, PP. 107-109, 2010.

[6] Pei Jiuling, Zhou Peng, Che Baochuan, Improving the Master Circuit Based on more Power Supersonic, Pipeline Technique and Equipment, No 2. PP. 51-52, 2008. 